\section{A MyoD-generated feed-forward circuit temporally patterns gene expression during skeletal muscle differentiation}

\author{
Bennett H. Penn, ${ }^{1,2}$ Donald A. Bergstrom, ${ }^{1,3}$ \\ F. Jeffrey Dilworth, ${ }^{1}$ Eyal Bengal, ${ }^{4,6}$ and \\ Stephen J. Tapscott ${ }^{1,2,5}$
}

\begin{abstract}
${ }^{1}$ Division of Human Biology, Fred Hutchinson Cancer Research Center, Seattle, Washington 98109, USA; ${ }^{2}$ Molecular and Cellular Biology Program, Fred Hutchinson Cancer Research Center and the University of Washington, Seattle, Washington 98109, USA; ${ }^{3}$ Department of Laboratory Medicine, University of Washington School of Medicine, Seattle, Washington 98195, USA; ${ }^{4}$ Department of Biochemistry, Rappaport Institute for Research in the Medical Sciences, Faculty of Medicine, Technion-Israel Institute of Technology, Haifa 31096, Israel
\end{abstract}

The development and differentiation of distinct cell types is achieved through the sequential expression of subsets of genes; yet, the molecular mechanisms that temporally pattern gene expression remain largely unknown. In skeletal myogenesis, gene expression is initiated by MyoD and includes the expression of specific Mef2 isoforms and activation of the p38 mitogen-activated protein kinase (MAPK) pathway. Here, we show that p38 activity facilitates MyoD and Mef2 binding at a subset of late-activated promoters, and the binding of Mef2D recruits Pol II. Most importantly, expression of late-activated genes can be shifted to the early stages of differentiation by precocious activation of $\mathrm{p} 38$ and expression of Mef2D, demonstrating that a MyoD-mediated feed-forward circuit temporally patterns gene expression.

Supplemental material is available at http://www.genesdev.org.

Received June 28, 2004; revised version accepted August 4, 2004.

Studies of transcriptional regulation at individual promoters have led to the general model that ordered recruitment of a combination of factors achieves gene-specific transcription (Cosma 2002). Global genomic analysis extends this model to show how complex transcriptional regulatory networks can emerge from the combinatorial regulation of individual genes and has identified classes of simple regulatory motifs, such as feed-forward loops and regulatory cascades (Fig. 1A) (Lee

[Keywords: MyoD; transcription; MAP kinase; Mef2; p38; feed-forward] Corresponding authors.

${ }^{5}$ E-MAIL stapscot@fhcrc.org; FAX (206) 667-6524.

${ }^{6}$ E-MAIL Bengal@tx.technion.ac.il; FAX 972-4-8535-773.

Article and publication are at http://www.genesdev.org/cgi/doi/10.1101/ gad. 1234304 et al. 2002; Milo et al. 2002; Shen-Orr et al. 2002). A current opportunity for developmental biology is to use these two approaches, promoter-specific molecular biology and systems network biology, to reveal the molecular events that temporally pattern multistage gene expression programs during development and cell differentiation.

The differentiation of skeletal muscle is a powerful system for studying the molecular regulation of a multistaged program of cell differentiation. Vertebrate myogenesis is regulated by the bHLH transcription factor MyoD, and its paralogs Myogenin, Myf-5, and MRF4. These act by heterodimerizing with E-proteins and binding CAnnTG recognition sites (Blackwell and Weintraub 1990). Genetic experiments have shown that MyoD or Myf-5 act as lineage-determination factors and Myogenin mediates terminal differentiation (for review, see Arnold and Braun 1996). When expressed in nonmuscle cell types in vitro, each of these factors is sufficient to drive differentiation into skeletal muscle and allows the process to be studied in molecular detail (Weintraub et al. 1989; Choi et al. 1990).

Studies of myogenesis have revealed a predictable temporal pattern of gene expression both in vivo and in vitro (Lin et al. 1994; Zhao et al. 2002). In our previous study, we characterized the timing of gene expression associated with muscle differentiation in a model system consisting of mouse embryonic fibroblasts (MEFs) expressing an inducible MyoD-Estrogen Receptor fusion protein (MyoD-ER), which allows synchronized skeletal muscle differentiation (Hollenberg et al. 1993; Bergstrom et al. 2002). Microarray analysis demonstrated that MyoD activity altered the expression of $\sim 5 \%$ of assayed genes in a temporally specific, multistaged response, with some genes activated within $6 \mathrm{~h}$, but others not activated until 2 d later (Bergstrom et al. 2002). Studies from other groups using myoblast cell lines differentiating in response to endogenous $\mathrm{MyoD}$ have made similar observations, both in regard to the scope of genes regulated and the multistaged response (Delgado et al. 2003; Tomczak et al. 2003). Chromatin immunoprecipitation (ChIP) assays revealed that MyoD directly regulates genes expressed throughout this program (Bergstrom et al. 2002), but it remains unclear how this single transcription factor can sequentially activate discrete sets of genes and establish a temporally patterned multistaged program of gene expression.

Factors not specific to skeletal muscle, such as Mef2 transcription factors and p38 mitogen-activated protein kinase (MAPK), also play a role in myogenesis. p38 activity increases during myogenesis and modulates terminal differentiation (Cuenda and Cohen 1999; Zetser et al. 1999; Li et al. 2000; Puri et al. 2000; Wu et al. 2000), and has been shown to directly phosphorylate the Mef2 transactivation domain (Han et al. 1997; Ornatsky et al. 1999; Zhao et al. 1999; Cox et al. 2003). Whereas these factors enhance the ability of MyoD to convert cells into muscle in vitro, the steps in transcriptional activation that they regulate have not been determined. Although these and other cofactors have been shown to enhance MyoD-mediated transcription at specific promoters, none have been shown to alter the developmental timing of gene expression; specifically, none have been shown 
A

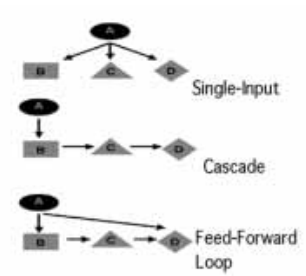

C

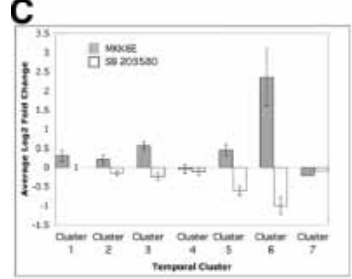

B Northern

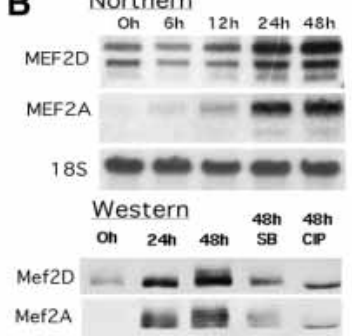

D

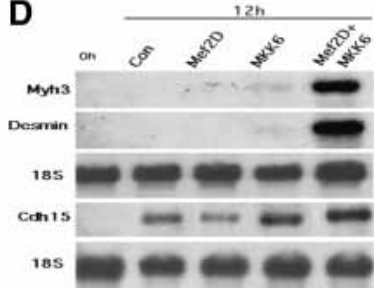

Figure 1. The p38 pathway and Mef2D are rate-limiting for latestage genes. (A) Examples of simple network motifs based on Lee et al. (2002). In a single-input motif, factor A directly regulates the three targets B, C, and D. The simple cascade depicts sequential activation with only gene $\mathrm{B}$ directly activated by $\mathrm{A}$. In the feedforward loop, A directly regulates each gene and sequential activation is achieved by requiring both $\mathrm{A}$ and $\mathrm{B}$ to express gene $\mathrm{C}$, and both $\mathrm{A}$ and $\mathrm{C}$ to express gene $\mathrm{D}$. $(B)$ MDER fibroblasts were induced to differentiate for varying times and analyzed by Northern and Western blot. (SB) Treatment of cells with SB203580; (CIP) phosphatase treatment prior to SDS-PAGE. $(C)$ Ratio of the average gene expression in each temporal cluster of MyoD-activated genes comparing cells constitutively expressing MKK6E or treated with SB203580 with the expression in untreated cells, demonstrating effects of p38 activity on clusters of genes activated at different stages of differentiation. Ratios are in $\log 2$ space; a value of 0 indicates no treatment change, a value of 1 indicates a twofold increase due to the treatment, and a value of -1 indicates a twofold reduction. Microarray expression data was generated from MDER cells induced to differentiate for $24 \mathrm{~h}$ with MKK6E-expressing virus or control virus, and analyzed together with previously generated data for control and SB203580-treated MDER cells (Bergstrom et al. 2002). Clusters 1-6 are the earliest to latest activated clusters, and cluster 7 is a transiently expressed group of genes (Bergstrom et al. 2002). Error bars represent standard error, except cluster 7 , where error bars are absent because only one gene from this cluster was affected. ANOVA p-value $<0.0001$ for both MKK6E and SB203580. Post-hoc testing indicated that cluster 6 differs significantly from the other clusters in response to MKK6E. (D) MDER cells were infected with the indicated retroviruses, induced to differentiate for $12 \mathrm{~h}$, and subjected to Northern analysis for late-stage genes Myh3 and Des and the early-stage gene Cdh15. Mef2 is the Mef2D isoform, Con is an empty control retrovirus.

to alter the temporal relationship among MyoD-activated genes.

Here, we analyze the molecular mechanisms that determine the temporal patterning of a subset of MyoD regulated genes. We show that Mef2 expression and p38 activity increase in response to MyoD. Binding of $\mathrm{MyoD}$ and Mef2 proteins at late-stage genes is temporally delayed until there is sufficient activity of $\mathrm{p} 38$. Transcription of this subset of promoters is further delayed until the Mef2D isoform joins the complex and then recruits Pol II. The expression of this subset of normally lateexpressed genes can be shifted to an earlier time relative to other MyoD targets by the precocious expression of Mef2D and an active p38 kinase. This demonstrates that a MyoD-generated feed-forward regulatory circuit, wherein factors induced by MyoD feed-forward to regu-

late MyoD activity at subsequent target genes, acts to temporally pattern the relative timing of gene expression during skeletal myogenesis.

\section{Results and Discussion}

p38 and Mef2D regulate the timing of a subset of genes during myogenesis

Our previously published microarray analysis using MyoD-ER showed early-stage induction of Mef2 isoforms and other factors that participate in the myogenic program (Bergstrom et al. 2002), consistent with numerous other studies in myoblast cell lines. Northern and Western analyses confirm that expression of both Mef2A and Mef2D increases in response to MyoD-ER (Fig. 1B). Retarded gel mobility of these proteins at later time points indicates increased phosphorylation, and treatment of cells with the p38 inhibitor SB203580 prevents the appearance of hyperphosphorylated Mef2, demonstrating a MyoD-induced activation of $\mathrm{p} 38$ and subsequent p38-dependent phosphorylation of Mef2A and Mef2D. In addition, in vitro kinase assays showed that both Mef2A and Mef2D are directly phosphorylated by p38 (data not shown).

Our prior microarray studies demonstrated that inhibition of p38 resulted in decreased expression of a subset of MyoD target genes. This effect was selective for genes normally expressed during the second day of the myogenic program, suggesting a potential role for $\mathrm{p} 38$ in regulating the timing of gene activation. To test this hypothesis, we assessed MyoD-induced gene expression in cells with precociously elevated p38 activity, achieved by the expression of MKK6E, an activated allele of the p38 upstream regulator (Han et al. 1996). Array analysis of genes expressed at $24 \mathrm{~h}$ following MyoD-ER induction identified a subset of genes that were more highly ex-
A

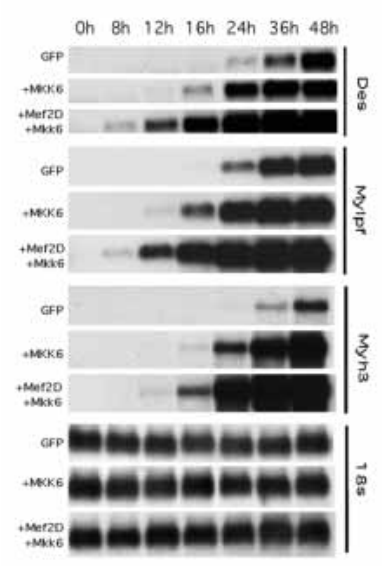

B

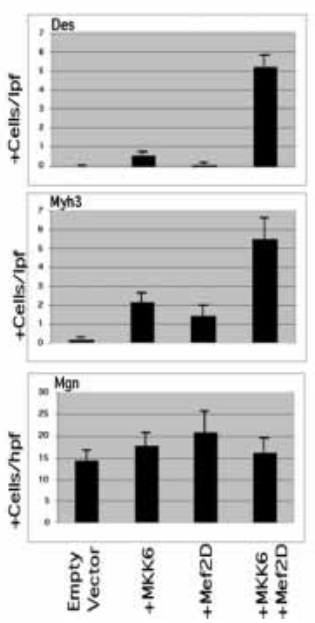

Figure 2. Mef2D and p38 are rate limiting for the activation of late-stage genes. (A) MDER cells were infected with MKK6E and/or Mef2D-expressing retrovirus as indicated, induced to differentiate for varying lengths of time, and analyzed by Northern blot with the indicated probes. GFP is used as a control retrovirus. $(B)$ MDER fibroblasts were transfected with the MKK6E and/or Mef2D retrovirus as indicated, induced to differentiate, and examined by immunofluorescence for the indicated late-stage (Myh3 and Des) and early-stage (Mgn) gene products. The bars indicate the average number of positive cells per field. 
pressed in the presence of active p38 (fold change $>2 \times$ and $q<0.10$; Supplementary Table 1). Compared with our previous study, the ability of MyoD to induce this set of genes was also inhibited by SB203580 ( $p=0.003)$, indicating that the level of p38 activity regulates MyoDmediated expression at this subset of promoters.

Additionally, when compared with the temporal profile of MyoD-regulated genes (Bergstrom et al. 2002), p38-responsive genes were normally expressed in the late stages of differentiation (Fig. 1C), suggesting the hypothesis that the MyoD-induced temporal increase of p38 activity might determine the timing, as well as the abundance of expression from this subset of promoters. Northern analysis showed that the p38 activation resulted in earlier expression of a number of late-stage p38regulated genes such as Desmin (Des), Myosin Light Chains 1 and 2 (Myl1, Mylpf), and embryonic Myosin Heavy Chain (Myh3) (Fig. 2A; data not shown), whereas the expression of early genes, such as Cdh15and Mef2A were not affected (Fig. 1D; data not shown). Pre-expressing Mef2D, one of the substrates of p38 relevant to skeletal myogenesis, had little effect on its own (Fig. 1D); however, when combined with active p38, expression of Mef2D resulted in still earlier gene expression (Figs. 1D, 2A). Single-cell analysis showed that Mef2D and p38 increase the number of cells expressing late-stage markers at an early time rather than simply increasing the abundance of expression from already expressing cells (Fig. 2B), whereas the number of cells expressing myogenin
$(M g n)$, a relatively early gene, was not affected. In the absence of MyoD activity, Mef2D and active p38 did not induce detectable target gene expression (Fig. 2A, time 0 ), indicating that these factors cooperate with MyoD to temporally pattern muscle gene expression. Together, these data demonstrate that MyoD regulates expression of Mef2 isoforms and activates the p38 pathway, and that these factors are rate limiting for the MyoD-dependent activation of some late-stage genes, suggesting that a feed-forward regulatory circuit, rather than a simple regulatory cascade, might temporally pattern MyoD-mediated gene expression.

\section{p38 regulates transcription-factor binding}

Next, we used ChIP assays to investigate the mechanisms by which the combination of p38, Mef2D, and MyoD regulate the expression of late-stage genes. ChIP, with an antiserum that recognizes multiple Mef2 isoforms (Mef2A, Mef2C, and Mef2D), demonstrated that p38 activation shifted the binding of Mef2 to an earlier time at Des, Myh3, and Myl1 (Fig. 3A), and that Mef2 binding required the presence of MyoD (Fig. 3B). The earlier binding of Mef2 was not due to increased Mef2 protein or DNA-binding affinity, because nuclear extracts from cells with an activated p38 kinase did not contain increased Mef2 DNA-binding activity in gelshift assays (data not shown). Activation of p38 also aug-
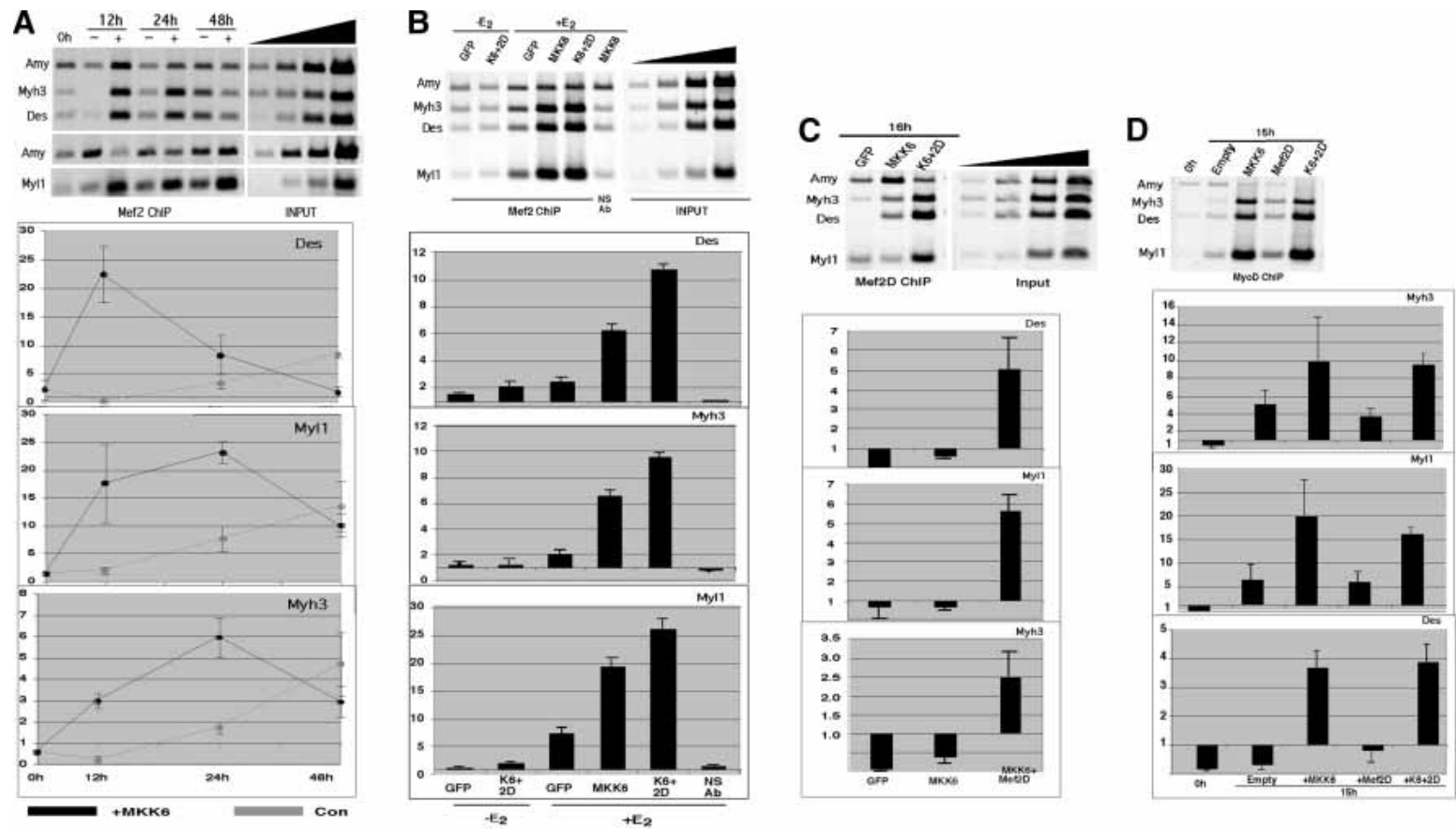

Figure 3. p38 regulates transcription factor recruitment. (A) MDER fibroblasts were either untreated $(0 \mathrm{~h})$ or infected with MKK6E retrovirus $(+)$ or empty vector control retrovirus (-) and induced to differentiate for 12,24 , or $48 \mathrm{~h}$. ChIP was performed with broad-specificity Mef2 antiserum, and multiplex PCR for the indicated promoters was performed with pancreatic amylase (Amy) as an internal control. Input chromatin was amplified over a 30-fold range to verify linearity of the assay. Graphs indicate IP promoter fold enrichment relative to input chromatin. Data for a representative ChIP are shown with error bars indicating S.E.M. for triplicate PCR. (B) Mef2 ChIP demonstrating that active MyoD is necessary for Mef2 binding, and that the broad-specificity Mef2 antisera identifies Mef2 binding both with and without exogenous Mef2D expression. (NS) Nonspecific IgG control ChIP; $\left(\mathrm{E}_{2}\right) \beta$-estradiol to induce MyoD activity. (C) ChIP using Mef2D monoclonal antibody shows Mef2D is not bound at early times in the absence of exogenous Mef2D. (GFP) A control retrovirus; (K6 + 2D or $6+\mathrm{D})$ a combination of the MKK6E and Mef2D retroviruses. $(D)$ ChIP using MyoD antisera shows p38 regulation of MyoD binding but no dependence on Mef2D. Input titration shown in C. (Empty) Control virus without effector gene. 
mented early MyoD binding at these promoters (Fig. 3D). Therefore, p38 activity regulates the formation of a MyoD and Mef2 transcription-factor complex at this set of late promoters.

Because Mef2D was rate limiting for the early expression of late-activated genes, it was surprising that constitutive expression of Mef2D, either with or without activated p38, had little effect on MyoD binding (Fig. 3D) and also failed to significantly increase total Mef2 binding (Fig. 3B); however, an antibody specific to Mef2D showed that this isoform was not present at early time points, and that constitutive expression of Mef2D significantly increased its binding in the presence of active p38 and MyoD (Fig. 3C). Therefore, the binding of the Mef2D isoform, but not total Mef2, correlates with MyoD-dependent transcriptional activation at this subset of late promoters. This suggests that only specific Mef2 isoforms can cooperate with MyoD to activate transcription at these promoters, and that the increasing abundance of the Mef2D isoform during differentiation fulfills a function subsequent to the binding of MyoD and other isoforms of Mef2.

\section{Mef2D and p38 regulate Pol II recruitment and progression at late promoters}

To understand the role of Mef2D in activating transcription of late-stage genes, we performed Pol II ChIP analysis. At an early time (12 h) following MyoD-induction, ChIP detected little Pol II recruitment at the Des, Myh3, or Myl1 promoters, and active p38 failed to augment it (Fig. 4A), despite inducing substantial binding of MyoD and total Mef2 (see Fig. 3 A,D). In contrast, constitutive expression of Mef2D, together with p38 and MyoD, resulted in early recruitment of Pol II to these muscle promoters (Fig. 4A). It is likely that this represents an active transcription complex because of the presence of Pol II with phospho-Ser 5 CTD (Fig. 4A). Because active p38 is required for stable Mef2 binding at these early time points, we cannot determine whether p38 has an additional role in Mef2D-mediated polymerase recruitment or phosphorylation.

At $16 \mathrm{~h}$ after MyoD-ER induction, when active p38 is sufficient to facilitate MyoD-mediated transcription without exogenous Mef2D (see Fig. 2A), possibly due to the presence of endogenous Mef2D at this later time, an active p38 increased the abundance of the active Ser 5-phosphorylated Pol II at the Des and Myh3 promoters and increased the abundance of the Pol II reaching downstream regions of these genes, but had only a marginal affect on Pol II recruitment (Fig. 4B). This suggests a second role for p38 in the progression of Pol II at some genes.

\section{MyoD initiates a feed-forward regulatory circuit}

We demonstrate that temporally patterned gene expression in a complex program of cell differentiation is achieved through a feed-forward mechanism. MyoD initiates the expression of specific Mef2 isoforms and activates the p38 MAPK pathway. p38 activity facilitates MyoD and Mef2 binding at genes expressed late in the myogenic program, and the binding of Mef2D recruits Pol II and correlates with the transcription of these genes. Most importantly, we show that expression of some late-stage genes can be shifted to the early stages of
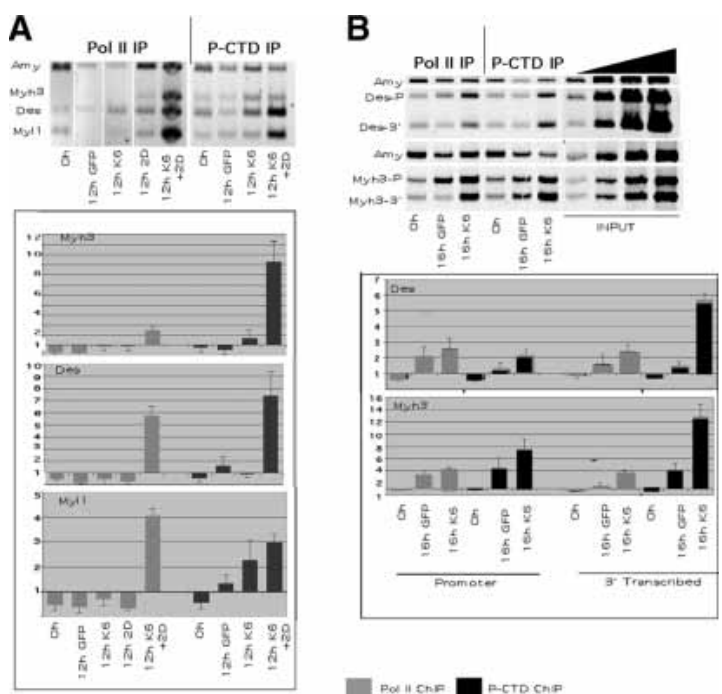

Figure 4. Mef2D and $\mathrm{p} 38$ regulate Pol II recruitment and progression. (A) MDER cells were transduced with control (GFP), MKK6E (K6), and/or Mef2D (2D) retroviruses, induced to differentiate $12 \mathrm{~h}$ and ChIP for Pol II, or P-CTD Pol II performed. The combination of Mef2D and p38 is associated with Pol II recruitment and phosphorylation. $(B)$ MDER cells were transduced with the indicated retroviruses and induced to differentiate for $16 \mathrm{~h}$. At this later time point when endogenous Mef2D and other muscle-specific Mef2 isoforms are accumulating, p38 activation induces polymerase progression and accumularion of P-CTD Pol II in the $3^{\prime}$ end of the gene. ChIP performed for Pol II and Ser 5-phosphorylated Pol II (P-CTD Pol II). Amplification of the gene promoter and a region in the 3 '-transcribed regions of the gene (exon 7 of desmin, $6.5 \mathrm{~kb}$ from the promoter, and exon 27 of MHC, $19 \mathrm{~kb}$ from the promoter) was performed. Titrations, internal control, and graphs are as previously indicated.

differentiation by precocious activation of p38 and expression of Mef2D, demonstrating that the timing of expression is programmed by an intrinsic delay while Mef2 isoforms and p38 activity accumulate, and substantiating the role of a transcriptional feed-forward circuit in temporally patterning gene expression. Because p38 and Mef2D cooperate with MyoD to regulate only a subset of late-stage genes, it is likely that additional sets of genes might require other MyoD-regulated intermediate factors.

Our study suggests two distinct roles of p38 kinase, as a rate limiting factor in the binding of Mef2 and MyoD, and a second role in facilitating phosphorylation and progression of Pol II. The role of p38 in facilitating the binding of MyoD and Mef2 is likely to be through an effect on chromatin, as it does not alter the binding of these factors in gel-shift assays (B. Penn and E. Bengal, unpubl.), and the recent demonstration that the p38 pathway targets the SWI/SNF complex to muscle loci through an interaction with MyoD might account for its effect on factor binding (Simone et al. 2004), although other mechanisms, such as histone phosphorylation (Saccani et al. 2002), might also effect factor binding. The role of p38 in facilitating Pol II phosphorylation and progression is likely to be through the phosphorylation of Mef2D, because prior studies have shown that p38 phosphorylation of the Mef2 activation domain greatly potentiates the transcriptional activity of Mef2 (Arnold and Braun 1996; Han et al. 1997; Ornatsky et al. 1999; Tamir and Bengal 2000). Our study shows that the Mef2D isoform 
is rate limiting for transcription at a subset of late promoters. This suggests that the Mef2D isoform has promoter-specific activities and that the relative abundance of Mef2 isoforms determines which subsets of promoters are actively transcribed.

In our previous study (Bergstrom et al. 2002), we showed that blocking p38 activity with SB203580 did not significantly delay the binding of MyoD to the Tncc2 gene, whereas, in this study, we show that precocious p38 activity induces precocious MyoD and Mef2 binding at other promoters. Together, these data indicate that p38 is sufficient to regulate MyoD binding, but is not necessary for MyoD binding at some promoters, perhaps because of parallel pathways. Our demonstration that p38 activity affects Pol II progression in addition to transcription factor binding at select promoters explains how SB203580 might inhibit MyoD-mediated expression of some genes at a step subsequent to MyoD binding.

The combination of microarray analysis of gene expression and molecular studies of transcription-factor binding are beginning to reveal the circuitry of gene expression networks. With these approaches, complex transcriptional programs can be deconstructed into a small number of simple regulatory motifs (see Fig. 1A) and mathematical modeling can illustrate the characteristics of each motif (Lee et al. 2002; Milo et al. 2002; Shen-Orr et al. 2002). Feed-forward circuits have an intrinsic time-delay, while intermediate factors accumulate and can have different characteristics that depend on the their several components (Mangan and Alon 2003). Our demonstration that skeletal muscle gene expression is temporally patterned by a MyoD-driven feedforward regulatory circuit provides a new framework for studying the molecular regulation of myogenesis and has implications for other complex programs of gene expression that unfold over time.

\section{Materials and methods}

\section{Cell culture}

Mouse fibroblasts (10T1/2 or Myf-5/MyoD null cells) were grown in $\mathrm{DME}+10 \%$ bovine calf serum (Hyclone), transduced with pBABEMyoD-ER, and selected in $1.4 \mu \mathrm{g} / \mathrm{mL}$ of puromycin (Sigma). HAhMKK6(E) in the pcLNCX (Naviaux et al. 1996) vector was packaged in $293 \mathrm{~T}$ cells with an ecotrophic envelope. hMef2D corresponds to sequence NM_005920.2. A 3'Flag epitope was added by PCR, cloned into pcLBABE (Naviaux et al. 1996) and packaged in 293T cells with an amphotropic envelope. Viral infections were done with $8 \mu \mathrm{g} / \mathrm{mL}$ of polybrene at an MOI of $5 \times$ for $24 \mathrm{~h}$, followed by exactly a 12 -h recovery. MyoD-ER was induced by culturing cells in DME with $0.5 \%$ horse serum, $10 \mu \mathrm{g} / \mathrm{mL}$ of insulin, $10 \mu \mathrm{g} / \mathrm{mL}$ of transferrin, and $10^{-7} \mathrm{M} \beta$-estradiol.

\section{ChIP, Western, immunofluorescence, and antibodies}

Antibodies used for ChIP were as follows: Mef2A (Santa Cruz), note that this antibody also recognizes Mef2C and Mef2D (B.H. Penn, unpubl.); Mef2D (BD Biosciences); Pol II (Santa Cruz); P-CTD Ser5 Pol II (Covance). Antibodies used for Western were as follows: Mef2D (BD Biosciences) and Mef2A (Santa Cruz). Antibodies used for immunofluorescence were as follows: Desmin (Dako), Myh3 (MF20), and Mgn (F5D). ChIP was performed as previously described, except that Quiaquick kits (QIAGEN) were used to purify DNA (Bergstrom et al. 2002). Multiplex PCR was performed in the linear range with an internal control. Products were detected by staining with SYBR Gold (Molecular Probes) and quantified using a Typhoon scanner (Molecular Dynamics).

PCR primers used in ChIP were Amy2+ (TCAGTTGTAATTCTCCT TGTACGG), Amy2- (CATTCCTTGGCAATATCAACC), Despro+ (AGC TCCTTGCCCTGTGAAAC), Despro- (GACGGAGCCCCTTAGCTG),
Desexon9+ (CAATGCAGCCTGGGGTGGATTTC), Desexon9- (CCC GGGTCTCAATGGTCTTGATCATC), Mylfenhancer+ (CAGCACACT GTCATGGGACCTAACC), Mylfenhancer- (GAGGAATTAGGCACCT GTTGTTTCGC), Mylfpro+ (GTCAGCTGTCACCAAACCAACC), Mylfpro(GAGCAGTGTGACTCGCTTGACC), Myh3pro+ (GCCCCCGAGG AGTTACAGAG), Myh3pro- (CAGAAAATGGCAGAGACAGCTTTG), Myh3exon27+ (TTGAGCACGAAGAAGCCAAGATTC), and Myh3exon27- (CGGAGGTGCTTGATGGTCTCTG).

For immunofluorescence studies, MDER cells were transfected with 2 $\mu g$ of pCLNC-HA-MKK6(E) and/or pCLBABE-Mef2D-Flag using Superfect (QIAGEN). Empty vector was used to normalize total DNA. Cells were induced $24 \mathrm{~h}$ after transfection and visualized by standard procedures.

Gene expression analysis

Northern Blot was performed by standard techniques with hybridization on Nytran $\mathrm{N}$ nylon membranes (Schleicher \& Schuell) using random prime generated cDNA probes. Microarray analysis was performed as previously described (Bergstrom et al. 2002).

Data analysis

All experimental conditions were represented by three independent replicates. Hybridized arrays were scanned using an Axon GenePix 4000 scanner and the GenePix 3.0 analysis program (Axon Instruments). Scanned data was normalized using the Lowess algorithm in the GeneSpring 6 Microarray Analysis Package (Silicon Genetics). A Lowess curve was fit to the log-intensity versus log-ratio plot. This curve was used to adjust the control value for each measurement. Normalized intensity ratios were transformed from linear to logarithmic space (base 2). Heteroscedastic Student's $t$-tests were performed comparing MyoD + MKK6E with MyoD + control and MyoD + SB203580 with MyoD + control. For the identification of MyoD-dependent genes significantly affected by the presence of MKK6E or SB203580, multiple test correction was performed by calculating a q-value for each feature from the distribution of p-values (Storey and Tibshirani 2003) using the R environment for statistical computing (RAqua for Mac OS X, compiled from R 1.8.0 by Stefano M. Iacus, Milan, Italy). Calculation of the q-value was restricted to the 143 features on the array that were significantly up-regulated after $24 \mathrm{~h}$ of MyoD activity [fold change $>2$ and $q<0.1$ in the MyoD $24 \mathrm{~h}$ vs. control $24 \mathrm{~h}$ data described previously (Bergstrom et al. 2002)] and informative for both the MKK6E and SB203580 analysis. Temporal cluster assignments were previously published (Bergstrom et al. 2002). All foldchange data presented in the Supplemental Material has been retransformed to linear space. The p-value for the overlap of MKK6E and SB203580 data was calculated using the hypergeometric distribution.

\section{Acknowledgments}

We thank Karen Seaver for assistance. This work was supported by NIH NIAMS AR 045113, B.H.P. was supported by the Medical Scientist Training Program (NIGMS 5-T32-07266), the Interdisciplinary Training Grant in Developmental Biology (NIH ST32HD07183), and a fellowship from the Poncin Foundation. F.J.D. is a Senior Research Fellow of the CIHR and is supported by an MDA Development Grant. E.B. received the ACSBI Fellowship from the UICC.

\section{References}

Arnold, H.H. and Braun, T. 1996. Targeted inactivation of myogenic factor genes reveals their role during mouse myogenesis: A review. Int. J. Dev. Biol. 40: 345-353.

Bergstrom, D.A., Penn, B.H., Strand, A., Perry, R.L., Rudnicki, M.A., and Tapscott, S.J. 2002. Promoter-specific regulation of MyoD binding and signal transduction cooperate to pattern gene expression. Mol. Cell. 9: 587-600.

Blackwell, T.K. and Weintraub, H. 1990. Differences and similarities in DNA-binding preferences of MyoD and E2A protein complexes revealed by binding site selection. Science 250: 1104-1110.

Choi, J., Costa, M.L., Mermelstein, C.S., Chagas, C., Holtzer, S., and Holtzer, H. 1990. MyoD converts primary dermal fibroblasts, chondroblasts, smooth muscle, and retinal pigmented epithelial cells into striated mononucleated myoblasts and multinucleated myotubes. Proc. Natl. Acad. Sci. 87: 7988-7992. 
Cosma, M.P. 2002. Ordered recruitment: Gene-specific mechanism of transcription activation. Mol. Cell. 10: 227-236.

Cox, D.M., Du, M., Marback, M., Yang, E.C., Chan, J., Siu, K.W., and McDermott, J.C. 2003. Phosphorylation motifs regulating the stability and function of myocyte enhancer factor 2A. J. Biol. Chem. 278: 15297-15303.

Cuenda, A. and Cohen, P. 1999. Stress-activated protein kinase-2/p38 and a rapamycin-sensitive pathway are required for $\mathrm{C} 2 \mathrm{C} 12$ myogenesis. J. Biol. Chem. 274: 4341-4346.

Delgado, I., Huang, X., Jones, S., Zhang, L., Hatcher, R., Gao, B., and Zhang, P. 2003. Dynamic gene expression during the onset of myoblast differentiation in vitro. Genomics 82: 109-121.

Han, J., Lee, J.D., Jiang, Y., Li, Z., Feng, L., and Ulevitch, R.J. 1996. Characterization of the structure and function of a novel MAP kinase kinase (MKK6). J. Biol. Chem. 271: 2886-2891.

Han, J., Jiang, Y., Li, Z., Kravchenko, V.V., and Ulevitch, R.J. 1997. Activation of the transcription factor MEF2C by the MAP kinase p38 in inflammation. Nature 386: 296-299.

Hollenberg, S.M., Cheng, P.F., and Weintraub, H. 1993. Use of a conditional MyoD transcription factor in studies of MyoD trans-activation and muscle determination. Proc. Nat1. Acad. Sci. 90: 8028-8032.

Lee, T.I., Rinaldi, N.J., Robert, F., Odom, D.T., Bar-Joseph, Z., Gerber, G.K., Hannett, N.M., Harbison, C.T., Thompson, C.M., Simon, I., et al. 2002. Transcriptional regulatory networks in Saccharomyces cerevisiae. Science 298: 799-804.

Li, Y., Jiang, B., Ensign, W.Y., Vogt, P.K., and Han, J. 2000. Myogenic differentiation requires signalling through both phosphatidylinositol 3-kinase and p38 MAP kinase. Cell. Signal. 12: 751-757.

Lin, Z., Lu, M.H., Schultheiss, T., Choi, J., Holtzer, S., DiLullo, C., Fischman, D.A., and Holtzer, H. 1994. Sequential appearance of musclespecific proteins in myoblasts as a function of time after cell division: Evidence for a conserved myoblast differentiation program in skeletal muscle. Cell. Motil. Cytoskeleton 29: 1-19.

Mangan, S. and Alon, U. 2003. Structure and function of the feed-forward loop network motif. Proc. Natl. Acad. Sci. 100: 11980-11985.

Milo, R., Shen-Orr, S., Itzkovitz, S., Kashtan, N., Chklovskii, D., and Alon, U. 2002. Network motifs: Simple building blocks of complex networks. Science 298: 824-827.

Naviaux, R.K., Costanzi, E., Haas, M., and Verma, I.M. 1996. The pCL vector system: Rapid production of helper-free, high-titer, recombinant retroviruses. J. Virol. 70: 5701-5705.

Ornatsky, O.I., Cox, D.M., Tangirala, P., Andreucci, J.J., Quinn, Z.A., Wrana, J.L., Prywes, R., Yu, Y.T., and McDermott, J.C. 1999. Posttranslational control of the MEF2A transcriptional regulatory protein. Nucleic Acids Res. 27: 2646-2654.

Puri, P.L., Wu, Z., Zhang, P., Wood, L.D., Bhakta, K.S., Han, J., Feramisco, J.R., Karin, M., and Wang, J.Y. 2000. Induction of terminal differentiation by constitutive activation of $\mathrm{p} 38$ MAP kinase in human rhabdomyosarcoma cells. Genes \& Dev. 14: 574-584.

Saccani, S., Pantano, S., and Natoli, G. 2002. p38-Dependent marking of inflammatory genes for increased NF-к B recruitment. Nat. Immunol. 3: 69-75.

Shen-Orr, S.S., Milo, R., Mangan, S., and Alon, U. 2002. Network motifs in the transcriptional regulation network of Escherichia coli. Nat. Genet. 31: 64-68.

Simone, C., Forcales, S.V., Hill, D.A., Imbalzano, A.N., Latella, L., and Puri, P.L. 2004. p38 pathway targets SWI-SNF chromatin-remodeling complex to muscle-specific loci. Nat. Genet. 36: 738-743

Storey, J.D. and Tibshirani, R. 2003. Statistical significance for genomewide studies. Proc. Nat1. Acad. Sci. 100: 9440-9445.

Tamir, Y. and Bengal, E. 2000. Phosphoinositide 3-kinase induces the transcriptional activity of MEF2 proteins during muscle differentiation. J. Biol. Chem. 275: 34424-34432.

Tomczak, K.K., Marinescu, V.D., Ramoni, M.F., Sanoudou, D., Montanaro, F., Han, M., Kunkel, L.M., Kohane, I.S., and Beggs, A.H. 2003. Expression profiling and identification of novel genes involved in myogenic differentiation. FASEB J. 18: 403-405.

Weintraub, H., Tapscott, S.J., Davis, R.L., Thayer, M.J., Adam, M.A., Lassar, A.B., and Miller, A.D. 1989. Activation of muscle-specific genes in pigment, nerve, fat, liver, and fibroblast cell lines by forced expression of MyoD. Proc. Nat1. Acad. Sci. 86: 5434-5438.

Wu, Z., Woodring, P.J., Bhakta, K.S., Tamura, K., Wen, F., Feramisco, J.R., Karin, M., Wang, J.Y., and Puri, P.L. 2000. p38 and extracellular sig- nal-regulated kinases regulate the myogenic program at multiple steps. Mol. Cell. Biol. 20: 3951-3964.

Zetser, A., Gredinger, E., and Bengal, E. 1999. p38 mitogen-activated protein kinase pathway promotes skeletal muscle differentiation. Participation of the Mef2c transcription factor. J. Biol. Chem. 274: 5193-5200.

Zhao, M., New, L., Kravchenko, V.V., Kato, Y., Gram, H., di Padova, F., Olson, E.N., Ulevitch, R.J., and Han, J. 1999. Regulation of the MEF2 family of transcription factors by p38. Mol. Cell. Biol. 19: 21-30.

Zhao, P., Iezzi, S., Carver, E., Dressman, D., Gridley, T., Sartorelli, V., and Hoffman, E.P. 2002. Slug is a novel downstream target of MyoD. Temporal profiling in muscle regeneration. J. Biol. Chem. 277: 30091-30101. 


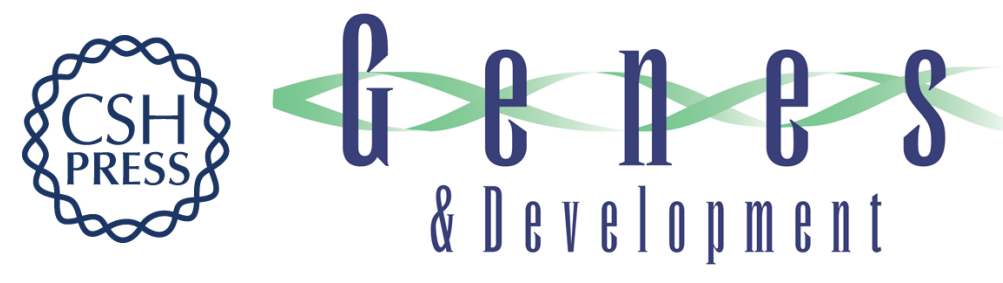

\section{A MyoD-generated feed-forward circuit temporally patterns gene expression during skeletal muscle differentiation}

Bennett H. Penn, Donald A. Bergstrom, F. Jeffrey Dilworth, et al.

Genes Dev. 2004, 18:

Access the most recent version at doi:10.1101/gad.1234304

Supplemental http://genesdev.cshlp.org/content/suppl/2004/09/10/18.19.2348.DC1
Material

References This article cites 30 articles, 18 of which can be accessed free at:

http://genesdev.cshlp.org/content/18/19/2348.full.html\#ref-list-1

License

Email Alerting

Receive free email alerts when new articles cite this article - sign up in the box at the top

Service

right corner of the article or click here.

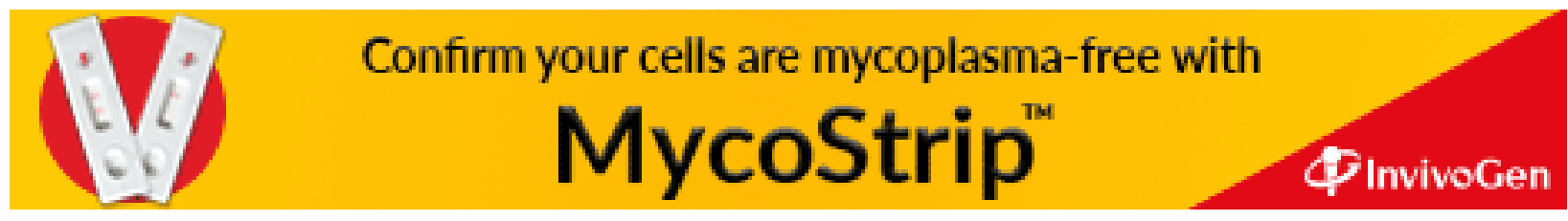

